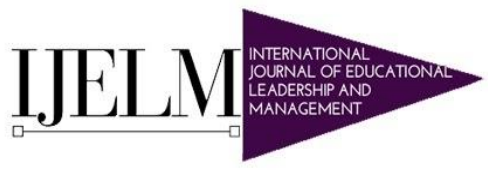

Hipatia Press

www.hipatiapress.com

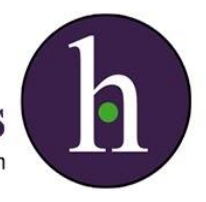

Instructions for authors, subscriptions and further details:

http://ijelm.hipatiapress.com

\title{
Formación para el liderazgo escolar: Impacto del Plan de formación de directores en Chile.
}

Richar Parra Robledo ${ }^{1}$ \& Carmen Ruiz Bueno ${ }^{1}$

1) Universitat Autònoma de Barcelona, Spain.

Date of publication: July $16^{\text {th }}, 2020$

Edition period: July 2019 - July 2020

To cite this article: Parra Robledo, R. \& Ruiz Bueno, C. (2020). Formación para el liderazgo escolar: Impacto del Plan de formación de directores en Chile. International Journal of Educational Leadership and Management. doi: 10.17583/ijelm.2020.4730

To link this article: http://dx.doi.org/10.17583/ijelm.2020.4730

\section{PLEASE SCROLL DOWN FOR ARTICLE}

The terms and conditions of use are related to the Open Journal System and to Creative Commons Attribution License (CCAL). 


\section{Formación para el liderazgo escolar: Impacto del Plan de formación de directores en Chile.}

Richar Parra Robledo

Universitat Autònoma de Barcelona

Spain
Carmen Ruiz Bueno

Universitat Autònoma de Barcelona

Spain

\section{Resumen}

El presente artículo tiene como objetivo evaluar el impacto de la formación de directores, desarrollado en el marco del Plan de formación en Chile desde el 2011 hasta la actualidad. La investigación es no experimental, ex post facto, con un muestro no probabilístico por conveniencia de 290 participantes de las acciones formativas realizadas a nivel de país. Para la recogida de la información, se diseñó un cuestionario, el cual presenta un índice elevado de consistencia interna global $(\alpha=0,980)$ entregado por el programa SPSS Statistics 25 . Entre los resultados, se identifica un alto impacto de la formación en el ámbito profesional, principalmente en las mujeres, en los niveles de aumento de la seguridad profesional y en la mejora de sus competencias directivas potenciando el impacto de la calidad y rendimiento de su trabajo en la organización escolar. Además, en este grupo, se aprecia un alto impacto en el ámbito organizacional, especialmente, en la promoción de una cultura de trabajo en equipo y en el aumento de las innovaciones educativas. Finalmente, se concluye que el mayor impacto de los programas formativos es en el propio aprendizaje y en el desarrollo profesional de las formadas y los formados.

Palabras claves: Evaluación de impacto, formación directiva, desarrollo profesional, organización escolar. 


\section{Training for school leadership: Impact of the Director Training Plan in Chile.}

Richar Parra Robledo

Universitat Autònoma de Barcelona

Spain
Carmen Ruiz Bueno

Universitat Autònoma de Barcelona Spain

\section{Abstract}

This article aims to assess the impact of the training of directors, developed in the framework of the Training Plan in Chile from 2011 to the present. The research is non-experimental, ex post facto, with a non-probabilistic sample for the convenience of 290 participants of the training actions carried out at the country level. For the collection of the information, a questionnaire was designed, which presents a high index of global internal consistency $(\alpha=0.980)$ delivered by the SPSS Statistics 25 program. Among the results, a high impact of training in the field is identified professional, mainly in women, in the levels of increased professional safety and in the improvement of their managerial competencies, enhancing the impact of the quality and performance of their work in the school organization. In addition, in this group, there is a high impact in the organizational field, especially in the promotion of a culture of teamwork and in the increase of educational innovations. Finally, it is concluded that the greatest impact of the training programs is on the learning itself and on the professional development of those trained and trained.

Keywords: Impact evaluation, management training, professional development, school organization. 


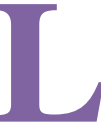

os resultados y las conclusiones de las investigaciones de organismos internacionales han recomendado fortalecer el liderazgo educativo, mediante políticas específicas (García-Garnica \& Caballero, 2019) dirigidas a mejorar los perfiles directivos, las funciones del cargo directivo y los programas de formación directiva. El objetivo es alinear las distintas políticas educativas con el claro propósito de fortalecer el liderazgo escolar (Cóndor-Quimbita \& Remache-Bunci, 2019; Ministerio de Educación [MINEDUC], 2016; Oficina Regional de Educación para América Latina y el Caribe [OREAL/UNESCO], 2016; Robinson, Hohepa \& Lloyd, 2009).

Las propuestas de abordaje de las sugerencias entregadas han sido transferidas en una heterogeneidad de experiencias entre los países de la Organización para la Cooperación y el Desarrollo Económicos (OCDE), las cuales se traducen en estructuras de soporte, tanto en la actualización de los perfiles directivos, diseño de una carrera directiva, certificación nacional para lideresas y líderes, creación de centros de liderazgos, profundización en los temas vinculados y la creación de programas de formación directiva con claras directrices (Cancino \& Vera, 2017; Centro de desarrollo del liderazgo educativo [CEDLE], 2018; García-Martínez \& Higueras-Rodríguez, 2018).

Considerando el contexto de cambio y de mejorar internacional, sumado a la contundencia de los estudios internacionales y nacionales (Cancino \& Vera, 2017; CEDLE, 2018; García-Garnica \& Caballero, 2019; OREAL/UNESCO, 2016), el Ministerio de Educación de Chile (MINEDUC) y el Centro de Perfeccionamiento, Experimentación e Investigación Pedagógica (CPEIP), dependiente del anterior, desarrollaron un Plan de formación de directores y directoras (PFD) (2011), inserto en la Política educativa de fortalecimiento del liderazgo directivo escolar desde el 2014 (MINEDUC, 2016).

Por cuanto es importante insistir en el objetivo y propósito del artículo, es decir insistir en la necesidad de evaluar el impacto de estos programas formativos como estrategias para la mejora continua, tanto de los programas 
de formación como de los centros educativos: su liderazgo, sus prácticas, por nombrar algunas.

\section{Plan de formación de directoras y directores}

El Plan de formación de directoras y directores (PFD) se estructura desde un posicionamiento centrado en lo pedagógico (Weinstein \& Hernández, 2015), con el propósito de desarrollar competencias y prácticas directivas requeridas para el ejercicio del cargo de director y directora (MINEDUC, 2011, 2016). En el transcurso del PFD, según datos públicos de las cohortes 2011-2016, se han ejecutado 104 acciones formativas, con un total de 4.277 participantes (CPEIP, 2018). El PFD ha sido evaluado en diferentes ocasiones. El CPEIP realizó un monitoreo constante y sistemático del programa, pero las evaluaciones de impacto fueron un elemento ausente en el interior del plan (CPEIP, 2018). En 2013, el Centro de Estudios del Ministerio de Educación (CEM) aplicó un cuestionario auto administrado, el cual fue calificado con bajos niveles de fiabilidad (CEM, 2013). En el 2014, el Plan se reformula en una propuesta más amplia, sistemática y estructura, llamada Política de fortalecimiento del liderazgo escolar, desde el mismo año, se inician las evaluaciones ex ante por parte del Ministerio de Desarrollo Social (Ministerio de Desarrollo Social [MDS], 2015).

En la actualidad, las investigaciones de evaluación del Plan de formación de directores y directoras son escasas y circunscritas a validaciones internas ex ante. Por tanto, consideramos imprescindible aumentar los vacíos en la literatura y, a la vez, incrementar los estudios sobre la transferencia y el impacto de la formación en los participantes, en las organizaciones escolares y en la sociedad.

\section{Evaluación de impacto de la formación}

Las evaluaciones de impacto se han comenzado a masificar, dado su potencial de visualización de los productos y resultados de los proyectos, programas o políticas públicas, en especial, por el reconocimiento de objetivos, propósitos y metas. Las evaluaciones de impacto desarrollan bases 
sólidas para la transparencia, aportando a determinar si se han logrado los resultados esperados (Consejo para la Transparencia, 2016; Dirección de Presupuestos [DIPRES], 2015). Además de lo anterior, se asume el poder intrínseco de las evaluaciones, el accountability i/o la rendición de cuentas a la sociedad civil (Consejo para la Transparencia, 2016; DIPRES, 2015; Feinstein, 2007; Gertler, Martínez, Premand, Rawlings \& Vermeersch, 2017). En el presente estudio, definimos operacionalmente la evaluación de impacto como la medición de los cambios producidos en una persona, organización o comunidad atribuibles a una acción formativa o programa formativo (Cano, 2016; Feixas, Fernández, Lagos, Quesada \& Sabaté, 2013; Melo, 2014; Centro Interamericano para el Desarrollo del Conocimiento en la Formación Profesional [OIT/Cinterfor], 2011; Pineda, 2011). Se desprende del constructo anterior la relación de causalidad (causa-efecto), entre los distintos impactos, los cambios producidos dependen de los objetivos de la formación y del foco de la medición, (Cejas, 2018; Feixas, Lagos, Fernández \& Sabaté, 2015; Martínez, Domínguez \& Infante, 2019; Pineda, 2011; Ramos, Meizoso \& Guerra, 2016).

Con el fin de contextualizar el estudio, debemos señalar el desarrollo de un modelo basado en la estructura general de la propuesta de evaluación de la formación de Kirkpatrick (1999) y de los factores de Baldwin y Ford (1988). La propuesta de evaluación desarrollada en la presente investigación tiene su génesis en la premisa de una evaluación para la mejora con un enfoque pedagógico. La estructura del modelo de evaluación del impacto de la formación se formula en cuatro dimensiones:

a) Condiciones personales. La dimensión se circunscribe a los factores individuales vinculados a las percepciones del participante, en temas focalizados a su desarrollo personal (Cejas, 2018; Gessler \& Hinrichs, 2015; Kirkpatrick, 1999; Naquin \& Holton, 2003; Park \& Wentling, 2007; Pineda, 2011; Rent, 2013).

b) Formación. La dimensión se enfocan en los factores de la estructura del plan de estudios de la formación, considerando el diseño curricular (Baldwin \& Ford, 1988; Gessler \& Hinrichs, 2015; Feixas et al., 2013; Kirkpatrick, 1999; Martínez, Domínguez \& Infante, 2019). 
c) Aplicación. La dimensión considera factores de la organización vinculados a la aplicación de la formación en el puesto de trabajo (Baldwin \& Ford, 1988; Cejas, 2018; Gracia-Pérez, Gil-Lacruz \& GilLacruz, 2017; Gessler \& Hinrichs, 2015; Kirkpatrick, 1999; Kleinert \& Pineda, 2018; López-Rodrigo, Feijoo-Cid, Novel-Martí \& LeyvaMoral, 2017; Martínez, Domínguez \& Infante, 2019; Tejada \& Ferrández, 2007; Tejada, Ferrández, Jurado, Más, Navío \& Ruiz, 2008).

d) Impacto. La dimensión se estructura en base a evaluar los impactos de la formación en los niveles profesionales, organizacionales y sociales (Cano, 2016; Gracia-Pérez, Gil-Lacruz \& Gil-Lacruz, 2017; Gertler et al., 2017; Martínez, Domínguez \& Infante, 2019; OIT/Cinterfor, 2011; Pineda, 2000).

El posicionamiento del estudio se desarrolla desde una perspectiva integral y sistémica de la evaluación de la formación. El enfoque de la investigación, considera el aprendizaje como un proceso de desarrollo personal, de cambio continuo, de mejora constante y paralelo a la formación (Gessler \& Hinrichs, 2015). Para tales efectos, las dimensiones de la medición tienen su extensión en el instrumento diseñado para valorar las percepciones de impacto de las acciones formativas del Plan de formación de directores y directoras.

\section{Método}

La investigación se desarrolló bajo el alero de un diseño no experimental, ex post facto (Hernández, 2018), con un muestro no probabilístico por conveniencia de 290 participantes de las acciones formativas realizadas en el marco del Plan de formación nacional desde el 2011 hasta el 2018. El objetivo del estudio es evaluar el impacto de la formación de directoras y directores en Chile. El procedimiento de elaboración del instrumento, se estructuro en etapas, las cuales se detallan a continuación:

La primera etapa fue el diseño y validación del instrumento de recogida de información. El proceso estuvo constituido por la elaboración del cuestionario electrónico, luego de una revisión exhaustiva de los 
instrumentos generados para evaluar de impacto de programas formativos de directores y directoras.

Posterior a la fase anterior, se solicitó la validación del instrumento por 14 jueces expertos en temas de detección de necesidades formativas y en la formación de líderes directivos escolares, para revisar su contenido y validez interna.

El juicio de experto/experta es una opinión informada de personas con trayectoria en el área de estudio, los cuales se encuentran cualificados profesional y académicamente. Los criterios de selección de los expertos/expertas fueron: (1) publicaciones en revista de divulgación académica, (2) proyectos de investigación en el campo y (3) presentación de ponencias o comunicaciones del tema en seminarios o congresos (EscobarPérez y Cuervo-Martínez, 2008).

Seleccionados los agentes validadores (expertos/expertas en el tema y metodólogos), se les envió un correo electrónico invitándolos a participar en la revisión crítica de la estructura, adecuación de las preguntas del cuestionario a los objetivos de la investigación, disposición de las dimensiones, ausencia o repetición de preguntas y extensión del instrumento. De las invitaciones generadas, 14 expertos/expertas examinaron el instrumento según tres criterios:

- Univocidad: hace referencia al nivel de precisión lingüística de la formulación del ítem, frente a la posible ambigüedad u otras interpretaciones del mismo.

- Pertinencia: hace referencia a la adecuación, idoneidad y / o relación del ítem con el objeto de estudio. Es decir, si el ítem es válido para aportar información de calidad sobre el objeto de estudio

- Importancia: hace referencia al peso específico del ítem al conjunto del cuestionario.

En la segunda etapa se ejecutaron reformulaciones requeridas por los expertos/expertas. Los ajustes en los ítems se operacionalizaron en preguntas más precisas y concretas. El orden de los ítems se reajustó en dimensiones 
puntuales, en una secuencia psicológica, ágil y amigable, para mejorar la comprensión de los participantes (García, 2003). El cuestionario final presenta una estructura interna de cuatro dimensiones con un total de 37 ítems.

Tabla 1.

Dimensiones del cuestionario

\begin{tabular}{|c|c|c|}
\hline Dimensión & Descripción & Ítems \\
\hline $\begin{array}{l}\text { I. Condicionantes } \\
\text { personales }\end{array}$ & $\begin{array}{l}\text { La dimensión se circunscribe a los factores individuales } \\
\text { vinculados a las percepciones del participante, en temas } \\
\text { focalizados a su desarrollo personal. }\end{array}$ & 9 \\
\hline II. Formación & $\begin{array}{l}\text { La dimensión se enfocan en los factores de la estructura del plan } \\
\text { de estudios de la formación, considerando el diseño curricular. }\end{array}$ & 10 \\
\hline III. Aplicación & $\begin{array}{l}\text { La dimensión considera factores de la organización, vinculados } \\
\text { a la aplicación de la formación y el desarrollo competencial en } \\
\text { el puesto de trabajo. }\end{array}$ & 7 \\
\hline IV. Impacto & $\begin{array}{l}\text { La dimensión se estructura en base a evaluar los impactos de la } \\
\text { formación en los niveles profesionales, organizacionales y } \\
\text { sociales. }\end{array}$ & 11 \\
\hline
\end{tabular}

La tercera etapa consistió en el envío del cuestionario a los correos electrónicos de los participantes por medio de la plataforma de Google forms. Terminado el proceso de recogida de los datos, se codificaron los resultados para su análisis en el programa SPSS Statistics 25. El paquete estadístico entregó una alfa de Cronbach de 0,980 al instrumento generado para evaluar el impacto de los programas de formación de directoras y directores, considerado un índice elevado de consistencia interna global. A continuación, se describen los resultados obtenidos. 


\section{Resultados}

En el siguiente apartado revisaremos los resultados del cuestionario de evaluación de impacto del Plan de formación de directoras y directores con una muestra de 290 participantes desde las cohortes 2011 al 2018. En el primer acercamiento estadístico, realizado por medio del programa estadístico SPSS Statistics 25, se describe la muestra productora de datos a través de medias generadas en cada subdimensión en sus respectivos ítems. En el segundo apartado, se profundiza en el análisis de los datos, comparando los grupos, según género, edad, dependencia, cargo y el año de participación. En la tercera parte, se revisan las correlaciones entre dimensiones y entre los impactos de la formación. Finalmente, en el cuarto apartado, se plantea la validación del instrumento, generando un modelo de la evaluación de impacto de programas formativos.

\section{Caracterización de la muestra}

La muestra participante en este estudio es de 290 casos, caracterizados de la siguiente manera. En género, se aprecia una distribución desigual, presentándose $61 \%$ de género femenino y $39 \%$ de género masculino. En referencia a la edad de los participantes de la muestra del Plan de formación de directores y directoras, la edad media es de 50 años, en un intervalo de una edad mínima de 29 años y una edad máxima de 66 años. Para la presente investigación, se recodificó la variable en tres niveles: novel (29-35 años), semi senior (26-50 años) y senior (51-66 años). En relación a la dependencia en la cual trabajan, la muestra, se encuentra focalizada con un $76 \%$ de participantes del sector municipal (público), seguido de un $24 \%$ de centros educativos particulares subvencionados o concertados. El cargo actual de los participantes presenta cuatro categorías, con una mayoría de los participantes en el cargo de director/a (81\%), la segunda mayoría, la obtiene las coordinadoras y los coordinadores $(13 \%)$. El porcentaje restante $(6 \%)$ se divide entre directivas y directivos y profesores/as, ambos con una representación del 3\%. Consultado sobre el año de participación en la formación, los resultados se distribuyen desde el 2011 al 2018, con una representación del $6,1 \%$ de la población total formados por el plan. 
Tabla 2.

Año de participación en la formación

\begin{tabular}{lrrrrrrrr}
\hline Cohorte & $\mathbf{2 0 1 1}$ & $\mathbf{2 0 1 2}$ & $\mathbf{2 0 1 3}$ & $\mathbf{2 0 1 4}$ & $\mathbf{2 0 1 5}$ & $\mathbf{2 0 1 6} / \mathbf{1 7}$ & $\mathbf{2 0 1 8}$ & Total \\
\hline Población & 778 & 807 & 626 & 816 & 501 & 749 & 458 & 4.735 \\
Muestra & 45 & 32 & 35 & 52 & 32 & 86 & 8 & 290 \\
Muestra \% & $5,8 \%$ & $4,0 \%$ & $5,6 \%$ & $6,4 \%$ & $6,4 \%$ & $11,5 \%$ & $1,7 \%$ & $6,1 \%$ \\
Dist. muestra & $15,5 \%$ & $11 \%$ & $12,1 \%$ & $17,9 \%$ & $11 \%$ & $29,7 \%$ & $2,8 \%$ & $100 \%$ \\
\hline
\end{tabular}

\section{1) Evaluación de Impacto de la formación.}

El instrumento diseñado para evaluar el impacto de la formación del Plan de formación de directoras y directores presenta una estructura interna de cuatro dimensiones con un total de 37 ítems con una escala de tipo Likert con una graduación de 1 a 4 ( 1 = poca/nunca/bajo; $4=$ mucha/siempre/alto). El análisis estadístico será ejecutado en cada dimensión.

Tabla 3.

Estructura de la Evaluación de Impacto de la Formación

\begin{tabular}{llc}
\hline Dimensión & Subdimensiones & Ítems \\
\hline I. Condicionantes personales & a) Expectativas & 1 - \\
& b) Contribución & $5-7$ \\
& c) Satisfacción & $8-9$ \\
II. Formación & a) Coherencia interna & $10-13$ \\
& b) Estrategias metodológicas & $14-19$ \\
III. Aplicación & a) Apoyo de la organización & $20-24$ \\
IV. Impacto & b) Apoyo de los equipos de trabajo & $25-26$ \\
& a) Impacto profesional & $27-31$ \\
& b) Impacto en la organización & $32-35$ \\
& c) Impacto social & $36-37$ \\
\hline
\end{tabular}


a) Dimensión Condicionantes personales. La dimensión consultaba a los participantes sobre la formación en tres subdimensiones: (a) expectativas, (b) contribución y (c) satisfacción. Las valoraciones más altas se focalizan en las expectativas de actualización de conocimientos profesionales (media=3,90), el intercambio de experiencia con otros profesionales (media $=3,68$ ). Los participantes perciben la mayor contribución de la formación en su desempeño profesional (media $=3,58$ ) y en el desarrollo de competencias directivas (media=3,59). Considerando una perspectiva global, la recomendación de formación puntúa (media=3,61). Los ítems con menor puntuación se enfocan en la generación de nuevas redes de contacto (media=3,13) y en mejorar expectativas laborales (media=3,14).

Tabla 4.

Valoración de la dimensión Condicionantes personales

\begin{tabular}{llc}
\hline Subdimensión / ítem & Media & S \\
\hline a. Expectativas & & \\
1. Mejorar expectativas laborales. & 3,14 & 0,98 \\
2. Generar nuevas redes de contacto. & 3,13 & 0,89 \\
3. Actualizar conocimientos profesionales. & 3,90 & 0,34 \\
4. Intercambiar de experiencias con otros profesionales. & 3,68 & 0,57 \\
b. Contribución & & \\
5. ... a su capacidad para aplicar el conocimiento. & 3,50 & 0,68 \\
6. ... al desarrollo de competencias directivas. & 3,59 & 0,65 \\
7. ... a su desempeño profesional. & 3,58 & 0,66 \\
c. Satisfacción & & \\
8. Se han cubierto sus expectativas con la formación. & 3,41 & 0,72 \\
9. Recomendaría la formación. & 3,61 & 0,67 \\
\hline
\end{tabular}

b) Dimensión Formación. La dimensión se enfoca en los factores de la estructura del plan de estudios de la formación, considerando la coherencia interna en el diseño curricular en dos elementos curriculares, como componentes teóricos fundamentales: (1) objetivos y (2) contenidos. La segunda dimensión, las estrategias metodológicas, se enfoca en un elemento curricular práctico y los diversos componentes que la articulan para lograr favorecer el aprendizaje transferible a los formados. Las valoraciones mejor 
puntuadas se centran en la correspondencia de los contenidos con los objetivos (media=3,55) y en la consecución de los objetivos (media=3,49). En cuanto a las estrategias metodológicas, las puntualizaciones más altas son el clima favorable para el aprendizaje promovido por los docentes (media $=3,66)$ y el fomento del trabajo cooperativo entre los participantes (media=3,63). Los ítems con menor valoración se puntúan en temas docentes, el primero, en el análisis de los nudos de aprendizajes no alcanzados (media $=3,35$ ) y en segundo, en la utilización del feedforward (media=3,40).

Tabla 5.

\section{Valoración de la dimensión Formación}

\begin{tabular}{lrc}
\hline Subdimensión / ítem & Media & S \\
\hline a. Coherencia interna & 3,49 & 0,65 \\
1. Se consiguieron los objetivos de la formación. & 3,43 & 0,71 \\
2. Los objetivos respondieron a sus necesidades. & 3,55 & 0,62 \\
3. Los contenidos correspondían a los objetivos de la formación. & 3,48 & 0,77 \\
4. La formación recibida es aplicable a otros contextos escolares. & & \\
b. Estrategia Metodológica & 3,50 & 0,65 \\
5. Los docentes demostraron idoneidad profesional durante su período & & \\
formativo. & 3,66 & 0,58 \\
6. Los docentes favorecieron un clima positivo para el aprendizaje. & 3,35 & 0,74 \\
7. Los docentes analizaron los nudos de aprendizaje de los contenidos no & & \\
alcanzados. & 3,40 & 0,75 \\
8. Los docentes utilizaron feedforward (sugerencias que ayudan a realizar & & \\
cambios positivos en su futuro) en su proceso formativo. & 3,63 & 0,61 \\
9. La formación promovió el trabajo cooperativo entre los participantes. & 3,57 & 0,63 \\
10. Los recursos de aprendizaje contribuyeron a la formación. & \\
\hline
\end{tabular}


c) Dimensión Aplicación. La dimensión recoge las percepciones de los participantes considerando variables de la organización vinculadas a la aplicación de la formación en el puesto de trabajo. Las valoraciones mejor puntuadas son el apoyo de los directivos (media=3,41) y las oportunidades laborales para aplicar la formación (media=3,39). Las puntuaciones más bajas las obtienen los ítems sobre la cultura de aprendizaje continuo $($ media $=3,16)$ y los procesos de innovación pedagógica $($ media=3,26).

Tabla 6.

Valoración de la dimensión Aplicación

\begin{tabular}{lcc}
\hline Subdimensión / ítem & Media & S \\
\hline a. Apoyo de la organización & & \\
1. La organización dispone de los recursos necesarios para aplicar los & 3,35 & 0,72 \\
conocimientos adquiridos en su formación. & & \\
2. El clima de la organización facilita la aplicabilidad de las competencias & 3,33 & 0,75 \\
$\quad$ adquiridas en la formación. & 3,16 & 0,86 \\
3. Existe una cultura de aprendizaje continuo en la organización. & 3,26 & 0,79 \\
4. La organización permite procesos de innovación pedagógica. & 3,39 & 0,80 \\
5. Independiente de su organización, existen oportunidades laborales para & & \\
aplicar la formación recibida. & & \\
b. Apoyo de los equipos de trabajo & 3,41 & 0,84 \\
6. Los directivos de su organización apoyan la aplicación de la formación. \\
7. Los profesionales de su organización contribuyen a la aplicación de la & 3,29 & 0,84 \\
\hline & & \\
\hline
\end{tabular}

d) Dimensión Impactos. Las valoraciones en la dimensión impactos, se encuentra dividida en tres niveles: (a) profesional, (b) organizacional y (c) social (Tabla 7). En el impacto profesional, detectamos las valoraciones más altas en la mejora del desempeño profesional (media $=3,73)$ y en el desarrollo de competencias directivas (media $=3,72$ ). En los impactos en la organización, la puntuación más alta es obtenida por el ítem, propiciar una cultura del trabajo en equipo (media=3,57). Las puntuaciones más bajas, en toda la dimensión, se aprecian en la mejora de los resultados académicos y en la mejora de la calidad de los aprendizajes de todos y todas las estudiantes, ambas con una valoración de (media=3,33). 
Tabla 7.

Valoración de la dimensión Impactos

\begin{tabular}{lrc}
\hline Subdimensión / ítem & Media & S \\
\hline a. Impacto profesional & & \\
1. Aumentar la seguridad profesional. & 3,57 & 0,69 \\
2. Mejorar su desarrollo profesional. & 3,73 & 0,54 \\
3. Desarrollar competencias directivas. & 3,72 & 0,61 \\
4. Mejorar la calidad de mi trabajo. & 3,61 & 0,66 \\
5. Mejorar el rendimiento en mi trabajo. & 3,57 & 0,68 \\
b. Impacto en la organización & & \\
6. Mejorar el clima escolar. & 3,40 & 0,68 \\
7. Mejorar los resultados académicos & 3,33 & 0,70 \\
8. Propiciar una cultura del trabajo en equipo. & 3,57 & 0,68 \\
9. Aumento de las innovaciones organizacionales. & 3,41 & 0,72 \\
c. Impacto social & & \\
10. Mejorar la calidad de los aprendizajes de todos y todas las estudiantes. & 3,33 \\
11. Vincular al establecimiento con organizaciones y actores de su & 3,38 & 0,71 \\
entorno. & & \\
\hline
\end{tabular}

\section{2) Comparación entre grupos.}

A fin de identificar diferencias significativas entre los grupos en la evaluación de impacto de la formación, se realizó una comparación entre muestras. Primero, se verificó la bondad de ajuste de la distribución, para lo cual se ejecutó la prueba de Kolmogorov-Smirnov con corrección de significación de Lilliefors. Al comprobar el nivel de significación obtenida $(0,00)$, los valores fueron menores a $\mathrm{p}<0,05$, por tanto, la distribución no es normal. En el segundo apartado, profundizamos en el análisis de los datos, comparando los grupos, según género, edad, dependencia, cargo y el año de participación.

a) Según género. Para analizar las diferencias entre dos categorías, en la variable género (femenino/masculino), se procedió a calcular la prueba U. de Mann-Withney. Al revisar los niveles de significación ( $\mathrm{p}<0,05$ ), las diferencias significativas se distinguen en la dimensión de impactos. Las 
mujeres participantes han respondido poseer un mayor impacto en el ámbito profesional (ítems 27, 28, 29 y 30) y en ámbito organizacional (ítems 34 y 35) a diferencia de los hombres.

b) Según edad. Para identificar la existencia de diferencias significativas entre las edades, se agrupó la variable continua (edad) en intervalos etarios y se aplicó la prueba de $\mathrm{H}$ de Kruskal Wallis. Al cotejar los niveles de significancia, las principales diferencias $(p<0,05)$, se identifican en las variables vinculadas a la dimensión de condicionantes personales (ítems 1, 5 y 7), en la dimensión formación (ítems 12 y 14) y la dimensión impactos (ítems 31, 32 y 36). Al revisar los resultados de la prueba post-hoc para ANOVA de 1 factor de Kruskal-Walis ( $\mathrm{k}$ muestras) por comparación múltiples, todo por parejas, apreciamos que el grupo de personas mayores de 55 años percibe una alta contribución de la formación en comparación a las personas menores de 34 (ítem 7). En la dimensión de los impactos, el grupo etario de participantes mayor de 55 años consideran que la formación ha generado un alto impacto en la mejora del clima escolar (32). Respecto a la mejora en la calidad de los aprendizajes de todos y todas las estudiantes (ítem 36), el segmento de participantes entre 35 y 44 años, percibe un mayor impacto respecto al grupo de personas mayores de 55 .

c) Según dependencia. A fin de comprobar la existencia de diferencias significativas entre las dependencias (municipal/particular subvencionado) en que trabajan los participantes de la formación, se ha procedido a calcular la U de Mann-Whitney para 2 muestras no relacionada. Los participantes provenientes del sector municipal (público) perciben un mayor impacto en las variables: mejora de las expectativas laborales (ítem 1), generación de nuevas redes de contacto (ítem 2), actualización de conocimientos profesionales (ítem 3) y vinculación del establecimiento con las organizaciones y actores de su entorno (ítem 37), a diferencia de los provenientes del ámbito educativo subvencionado (concertado).

d) Según cargo. Para identificar en qué cargo se presentan las diferencias significativas, se efectuó la prueba post-hoc para ANOVA de 1 factor de Kruskal-Walis. Al analizar los valores entre las muestras I y II, se distinguen 27 diferencias significativas $(\mathrm{p}<0,05)$, divididas en cuatro grupos: 
1) Los directores y directoras perciben un mayor impacto de la formación que los profesionales sin cargo, en las dimensiones condiciones personales (ítems 5 - 8), en la dimensión de aplicación (ítems 20 - 26) y en la dimensión impactos (ítems 27 - 31 y 35);

2) Los coordinadores y coordinadoras identifican un mayor impacto de la formación en la contribución al desarrollo de competencias (ítem 6), en la seguridad profesional (ítem 27), en la calidad de su trabajo (ítem 30) y en el aumento de innovaciones organizacionales (ítem 35), al ser comparado con los profesionales sin cargo;

3) Los equipos directivos aprecian un mayor impacto de la formación que los profesionales sin cargo, en el desempeño profesional (ítem 7), en el aumento de la seguridad profesional (ítem 27) y en las competencias directivas (ítem 29);

4) Los directores y directoras perciben un mayor impacto de la formación en la contribución al desempeño profesional (ítem 7), en las expectativas sobre la formación (ítem 8) y las oportunidades laborales para aplicar (ítem 28) al compararlo con los coordinadores.

e) Según año de participación. Para la comparación de los resultados, según la categoría año de participación en la formación, se han generado dos grupos (I. 2011-2014; II. 2015-2018). Para el análisis, se aplicó la prueba no paramétrica U. de Mann-Withney. Al revisar los niveles de significación ( $\mathrm{p}$ $<0,05)$, se aprecia, en todos los casos analizados (26 ítems), el grupo I perciben un mayor impacto de la formación al comparar con el grupo II. 


\section{3) Relaciones entre variables.}

Considerando las posibles correlaciones entre las dimensiones, los impactos, se ha decidido aplicar el estadístico de rho de Spearman para identificar las relaciones con significación $(\mathrm{p}<0,01)$.

a) Correlaciones entre dimensiones. Al analizar los resultados de la correlación entre las dimensiones de la evaluación de impacto de la formación (Tabla 8), se identifica la mayor correlación entre la dimensión formación y la dimensión impactos, con valor de 0,631. La menor correlación se ha obtenido entre la dimensión condicionantes personales y la dimensión aplicación, con un valor de 0,398.

Tabla 8.

Correlación entre dimensiones

\begin{tabular}{|c|c|c|c|c|}
\hline Correlaciones & I. Cond. personales & II. Formación & III. Aplicación & IV. Impactos \\
\hline I. Cond. personales & & & & \\
\hline II. Formación & $0,551^{* *}$ & & & \\
\hline III. Aplicación & $0,398^{* *}$ & $0,509^{* *}$ & & \\
\hline IV. Impactos & $0,605^{* *}$ & $0,631^{* *}$ & $0,539^{* *}$ & \\
\hline
\end{tabular}

Respecto a las correlaciones entre las dimensiones, se identifica una relación positiva moderada: 1) Entre las condicionantes personales y la formación $(r=0,551), 2)$ Entre las condiciones personales y los impactos $(r=0,605)$, y 3) entre la formación y los impactos $(\mathrm{r}=0,631)$.

b) Correlación entre impactos. Al examinar las correlaciones entre los impactos de formación con el coeficiente de rho de Spearman (Tabla 9), se aprecia una significación inferior a 0,01 , en todos los niveles considerados en el instrumento (profesional, organizacional y social). La mayor correlación se identifica en el impacto entre la mejora del desarrollo profesional (ítem 28) y el desarrollo de competencias directivas (ítem 29), con 0,862. La menor relación se distingue entre el aumento de la seguridad profesional (ítem 27) y la mejora de los resultados (ítem 33), con una correlación de 0,311 . 
Tabla 9.

Correlación entre impactos

\begin{tabular}{|c|c|c|c|c|c|c|c|c|c|c|c|}
\hline \multirow[b]{2}{*}{ ítem } & \multicolumn{5}{|c|}{ Profesional } & \multicolumn{4}{|c|}{ Organizacional } & \multicolumn{2}{|c|}{ Social } \\
\hline & 27 & 28 & 29 & 30 & 31 & 32 & 33 & 34 & 35 & 36 & 37 \\
\hline 27 & & & & & & & & & & & \\
\hline 28 & $0,732 *$ & & & & & & & & & & \\
\hline 29 & $0,656^{*}$ & $0,862^{*}$ & & & & & & & & & \\
\hline 30 & $0,641^{*}$ & $0,743^{*}$ & $0,731 *$ & & & & & & & & \\
\hline 31 & $0,568 *$ & $0,687^{*}$ & $0,648^{*}$ & $0,769^{*}$ & & & & & & & \\
\hline 32 & $0,338^{*}$ & $0,466^{*}$ & $0,460 *$ & $0,461^{*}$ & $0,477 *$ & & & & & & \\
\hline 33 & $0,311^{*}$ & $0,434 *$ & $0,455^{*}$ & $0,482 *$ & $0,471^{*}$ & $0,528^{*}$ & & & & & \\
\hline 34 & $0,379^{*}$ & $0,518^{*}$ & $0,546^{*}$ & $0,487 *$ & $0,430 *$ & $0,569^{*}$ & $0,580 *$ & & & & \\
\hline 35 & $0,356^{*}$ & $0,489^{*}$ & $0,473^{*}$ & $0,497 *$ & $0,442 *$ & $0,526^{*}$ & $0,532 *$ & $0,670^{*}$ & & & \\
\hline 36 & $0,322 *$ & $0,411^{*}$ & $0,410^{*}$ & $0,461^{*}$ & $0,466^{*}$ & $0,483^{*}$ & $0,628^{*}$ & $0,520^{*}$ & $0,526^{*}$ & & \\
\hline 37 & $0,343 *$ & $0,439 *$ & $0,415^{*}$ & $0,476^{*}$ & $0,474 *$ & $0,500^{*}$ & $0,456^{*}$ & $0,483^{*}$ & $0,532 *$ & $0,552 *$ & \\
\hline
\end{tabular}

* . La correlación es significativa en nivel 0,01 (bilateral).

En referencia a la correlación entre los impactos, se aprecia una relación positiva fuerte: 1) Entre el desarrollo profesional y el incremento de las competencias directivas $(\mathrm{r}=0,862)$ y 2 ) Entre la mejora de la calidad del trabajo y el aumento en el rendimiento laboral $(\mathrm{r}=0,769)$.

\section{4) Validación del modelo.}

A fin de desarrollar una validación del modelo para la evaluación del impacto de la formación, se han considerado tres aspectos fundamentales: (a) Análisis factorial de los ítems, (b) Fiabilidad y (c) Regresión múltiple.

a) Análisis factorial. Para validar el cuestionario, se desarrolló una validación de constructo mediante el procedimiento de análisis factorial exploratorio por medio del programa estadístico SPSS Statistics 25. El primer paso fue excluir aquellas variables consideradas resultados de la formación, en este caso, las vinculadas a las dimensiones condicionantes personales, formación, aplicación e impactos, las cuales fueron sometidas al análisis de fiabilidad. El segundo paso fue seleccionar el método máxima verosimilitud, procediendo al análisis con una rotación de factores Varimax (rotación ortogonal), con valor Eigen mayor a la unidad y con el valor 
mínimo de los coeficientes a 0,40. La prueba de esfericidad de Barlett y la KMO indicaron la factibilidad de un modelo adecuado de análisis (KMO de 0,922 y una significación de Barlett de 0,000). Posterior a una revisión de los factores y análisis de ellos, se identificó la presencia de 6 factores. Concluido el análisis, el modelo obtenido explica el 60,7\% de la varianza, con 6 factores y con un total de 24 ítems de los 26 ítems al comenzar el análisis. Los factores resultantes son: (1) apoyo docente, (2) apoyo de la organización, (3) coherencia interna de la formación, (4) percepción de contribución, (5) expectativas de la formación y (6) apoyo de los equipos de trabajo.

b) Fiabilidad. La fiabilidad del instrumento fue determinada mediante el alfa de Cronbach, considerando puntuaciones mayores a 0,70. Los valores del alfa tipificado son: factores $(0,922)$, condicionantes personales $(0,700)$, formación $(0,926)$, aplicación $(0,910)$. En cuanto a los impactos, se decidió analizar cada resultado por separado, por tanto, fragmentamos, en impacto profesional $(0,930)$, en impacto organizacional $(0,868)$ y en impacto social $(0,742)$.

c) Regresión múltiple. El último procedimiento realizado fue la regresión múltiple, para determinar en qué grado los factores y las variables consideradas poseen propiedades predictivas. El procedimiento ejecutado fue seis regresiones múltiples, considerando los seis factores y su relación con cada dimensión; primero de forma exploratoria, con el método paso a paso y, posteriormente, con el método introducción. En todos los análisis de los resultados finales con el método introducción, se revisó el cumplimiento de supuestos: 1) independencia, 2) la no existencia de multicolirealidad (FIV $<$ 10; media FIV = 1) y 3) la independencia de residuos (Test de Durbin Watson =2).

Los resultados del primer análisis predictivo de los factores personales otorgan un valor de $\mathrm{R}^{2}$ de 0,811 que al ser corregida por efectos muéstrales $\mathrm{y}$ por las propias variables independientes baja a 0,809. El error típico de la estimación tiene un valor de 0,166. Esto significa que el modelo explica el $80,9 \%$ de la varianza. El modelo de regresión uno, se formuló de la siguiente manera: Condicionantes personales $=3,506+0,262$ expectativas de la 
formación $+0,232$ percepción de contribución $+0,145$ coherencia interna de la formación.

El segundo análisis predictivo relacionó los factores y las condicionantes personales con la formación. En este caso, los factores son 1, 2 y 3, y las condicionantes personales explican el $97 \%$ de la varianza. Los valores de $\mathrm{R}^{2}$ corregida es de 0,970, el error típico ha alcanzado un valor de 0,090. El modelo se formula de la siguiente manera: Formación $=2,505+0,394$ apoyo docente $+0,285$ condicionantes personales $+0,247$ coherencia interna de la formación $+0,078$ apoyo de la organización.

El tercer análisis predictorio, corresponde a los factores, las condiciones personales y la formación respecto a la aplicación. Los factores vinculados son 2 y 6 , y la formación explica el $97,5 \%$ de la varianza. El $\mathrm{R}^{2}$ es de un valor 0,976, el $\mathrm{R}^{2}$ corregido es de 0,975. El error típico de 0,101. La configura de la fórmula es: Aplicación $=2,180+0,577$ apoyo de la organización + 0,346 formación $+0,141$ apoyo de los equipos de trabajo.

Considerando la prospectiva del modelo, es necesario fragmentar las áreas de impacto de la evaluación de la formación. Desde esta perspectiva, es importante plantearnos en tres ámbitos de la medición (profesional, organizacional y social). En referencia al impacto profesional, se ejecutó un análisis multivariable con los seis factores, las condicionantes personales, la formación y la aplicación. El modelo incluye el factor 4, la formación y la aplicación, explicando el 59,7\% de la varianza. El valor del $\mathrm{R}^{2}$ es de 0,601 y de la $\mathrm{R}^{2}$ corregida de 0,597 , con un error típico de 0,359 . La formulación resultante es: Impacto profesional $=1,112+0,511$ formación $+0,223$ aplicación $+0,192$ percepción de contribución.

El análisis del impacto organizacional se desarrolló en los seis factores, las condicionantes personales, la formación, la aplicación y el impacto profesional. La correspondencia del modelo resultante es que el impacto profesional, la formación y la aplicación, explican el 53,1\% de la varianza. El valor de $\mathrm{R}^{2}$ es de 0,536 , el de $\mathrm{R}^{2}$ corregido $0,531 \mathrm{y}$ el error típico es de 0,403 . El modelo se formuló de la siguiente manera: Impacto organizacional $=0,332+0,482$ impacto profesional $+0,224$ formación $+0,168$ aplicación 
El último análisis se realizó considerando los seis factores, las condicionantes personales, la formación, la aplicación, el impacto profesional y el impacto organizacional. El modelo se configura con los factores 1 y 5 , el impacto organizacional y la aplicación, explicando el $59,6 \%$ de la varianza. Los valores de $\mathrm{R}^{2}$ es de 0,602 , el de $\mathrm{R}^{2}$ corregida es de 0,596 y el error típico es de 0,405 . La formula es la siguiente: Impacto social $=0,682+0,682$ impacto organizacional $+0,118$ apoyo docente $+0,100$ aplicación $+0,058$ expectativa de la formación.

A fin de generar un apoyo visual del modelo resultante del análisis predictivo y sus relaciones, se diseñó la siguiente propuesta (Figura 1).

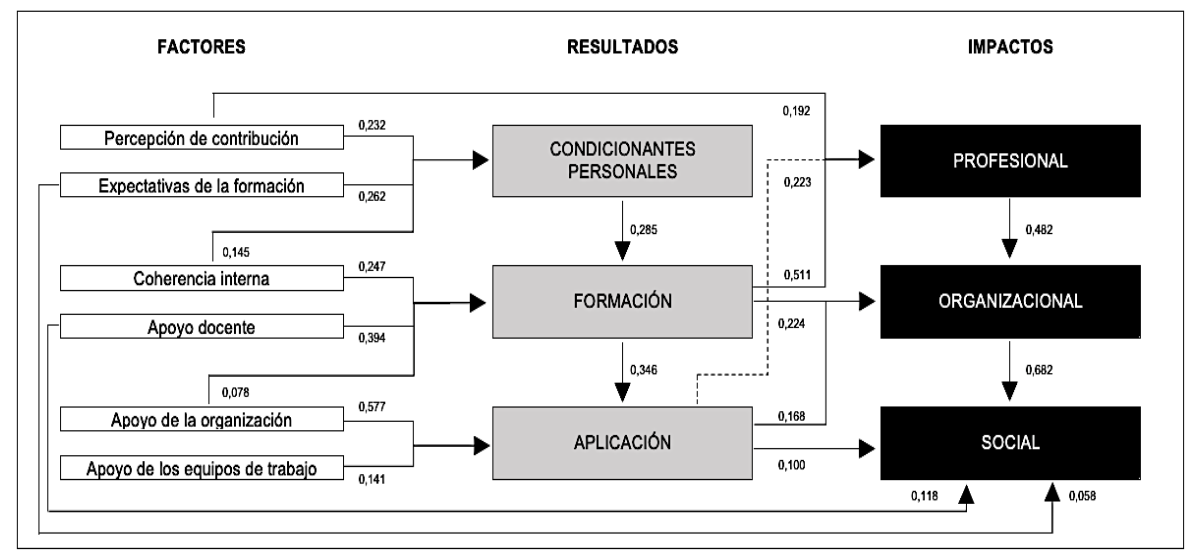

Figura 1. Modelo de factores de impacto

\section{Discusión y Conclusiones}

La evaluación de impacto de la formación de directores y directoras es un proceso evaluación de los cambios producidos por las relaciones causales en niveles personales, profesionales y organizacionales, atribuibles a una acción formativa. Por tanto, las conclusiones finales las desarrollaremos generando una visión sistémica desde una perspectiva integradora del proceso evaluativo, considerando un análisis en cada nivel. 
Examinando las valoraciones positivas en la dimensión de los condicionantes personales, podemos concluir la existencia de una fuerte contribución de la formación en el desempeño profesional y el desarrollo de competencias directivas, concordando con la investigación de Feixas et al. (2013), Rent (2013) y Tejada et al. (2008). En sí, las condicionantes personales pueden condicionar los aprendizajes adquiridos en la formación Gessler y Hinrichs (2015) y Cejas (2018). En cuanto a las expectativas, las valoraciones más altas se focalizan en la actualización de conocimientos profesionales y en el intercambio de experiencia con otros profesionales; considerando una valoración global de las acciones formativas, el $90 \%$ de los y las participantes recomiendan la formación.

Al evaluar la formación, los y las participantes valoran con mayor puntuación, la correspondencia entre los contenidos, los objetivos y su consecución, ofreciendo mayores posibilidades de aplicación de la formación en la organización (Cejas, 2018; Feixas et al., 2013; Gessler \& Hinrichs, 2015; Kirkpatrick, 1999; Pineda, 2011; Rent, 2013). Respecto a las estrategias metodológicas, las puntualizaciones más altas son el clima favorable para el aprendizaje favorecido por los docentes y la promoción del trabajo cooperativo entre los participantes. Cabe señalar, que los factores con menor valoración se puntúan en temas docentes, el primero, en el análisis de los nudos de aprendizajes no alcanzados y en segundo, en la utilización del feedforward (Feixas et al., 2013; Tomás-Folch \& Durán-Bellonch, 2017). Por tanto, podemos afirmar la existencia de una influencia paralela entre las estrategias metodológicas y el desarrollo de competencias directivas, cuanto mayor sea el clima favorable y el trabajo cooperativo mayor será el grado de competencias, ofreciendo mayores posibilidades de preparación, desarrollo y ejercicio en el puesto de trabajo y en la organización escolar (LópezRodrigo, Feijoo-Cid, Novel-Martí \& Leyva-Moral, 2017). El estudio de Martínez, Domínguez \& Infante (2019), confirman el hallazgo anterior, corroborando la mutua influencia entre los factores y una interrelación entre ambas dimensiones.

La investigación de Gilbert y Gibbs (1999) reconoce la importancia de la práctica reflexiva en los aprendizaje y desempeños de los y las participantes. 
Las estrategias metodológicas de la acción formativa están vinculadas a las metodologías analíticas deductivas, las cuales resultan pertinentes, al considerar a los y las participantes líderes escolares, los cuales deberán ser capaces de rediseñar y establecer una dirección en la organización escolar desde una perspectiva de cambio para la mejora.

Respecto a la aplicación de la formación en el puesto de trabajo, las y los participantes perciben con una alta valoración el apoyo de los directivos y las directivas (Baldwin \& Ford, 1988; Gessler \& Hinrichs, 2015; Holton, 1996; Kleinert \& Pineda, 2018) y las oportunidades laborales para aplicar (Kirkpatrick, 1999; Pineda, 2000). Sumado a lo anterior, el 83\% de los formados consideran al clima de la organización un facilitador de la transferencia de competencias adquiridas, por ende, cuanto más positivo sea el clima de la organización mayor será la seguridad del profesional para aplicar el aprendizaje en su puesto de trabajo. Los factores con menor valoración están vinculados a la baja percepción de una cultura de aprendizaje continuo, la falta de soporte en los procesos de innovación pedagógica y al poco apoyo de los profesionales en la transferencia de la formación a la organización (Baldwin \& Ford, 1988; Gessler \& Hinrichs, 2015).

En cuanto a los impactos, se identifica un mayor impacto de la formación en el ámbito profesional, en especial, en la mejora del desarrollo profesional y en las competencias directivas (Gracia-Pérez, Gil-Lacruz \& Gil-Lacruz, 2017). El mayor impacto en la organización se aprecia en motivar a los colaboradores para que sea posible una cultura del trabajo en equipo. Los menores impactos de la formación se aprecian, en la mejora de los resultados académicos, en la mejora de la calidad de los aprendizajes de todos y todas las estudiantes, y en la vinculación del establecimiento con organizaciones y actores de su entorno.

En referencia a los hallazgos en las comparaciones entre grupos, podemos afirmar que las mujeres participantes en la formación perciben un mayor impacto en el aumento de la seguridad profesional, en el desarrollo profesional y en las competencias directivas, potenciando la calidad y el 
rendimiento en el trabajo al compararlo con los hombres. Sumado a lo anterior, las profesionales identifican un alto impacto organizacional, en cuanto facilitan una cultura del trabajo en equipo y en el aumento de las innovaciones educativas a diferencia de los hombres.

Los mayores de 55 años consideran que la formación ha generado un alto impacto en la mejora del clima escolar. Respecto a la mejora en la calidad de los aprendizajes de todos y todas las estudiantes, el segmento de participantes entre 35 y 44 años, señalan un mayor impacto.

Los participantes provenientes del sector público aprecian un mayor impacto en la mejora de las expectativas laborales, en la generación de nuevas redes de contacto, en la actualización de conocimientos profesionales y en vincular el establecimiento con las organizaciones y actores de su entorno a diferencia de los participantes provenientes del ámbito subvencionado.

Finalmente podemos concluir, que el desarrollo profesional y competencial en los y las participantes se produce de forma paralela y no secuencial entre la formación y su aplicación en la organización, generando una relación entre ambas. Además, se aprecia que el mayor de impacto de los programas formativos es en el propio aprendizaje de los y las participantes y en su desarrollo profesional.

\section{Limitaciones del estudio}

Las limitaciones del estudio se identifican en la falta de técnicas y estrategias cualitativas para profundizar en las percepciones de los y las participantes sobre la formación directiva. Además, sería pertinente ampliar los agentes para desarrollar una triangulación de fuentes, considerando valoraciones de los colaboradores y colaboradoras de las organizaciones escolar, con el propósito de medir el impacto de la formación directiva en las prácticas profesionales docentes. También es relevante aumentar las investigaciones sobre los impactos sociocomunitarios de la formación en los centros escolares como referente de mejora en la calidad educativa. 
Parra \& Ruiz - Formación para el liderazgo escolar

\section{Referencias}

Baldwin, T., \& Ford, J. (1988). Transfer of training: A review and directions for future research. Personnel Psychology, 41, 63-105. doi: http://doi.org/cq3btj

Cancino, V., \& Vera, L. (2017). Políticas educativas de fortalecimiento del liderazgo directivo: desafíos para Chile en un análisis comparado con países OCDE. Avaliação e Políticas Públicas em Educação, 25(94), 26-58. doi: http://doi.org/c5nq

Cano, E. (2016). Factores favorecedores y obstaculizadores de la transferencia de la formación del profesorado en educación superior. REICE. Revista Iberoamericana sobre Calidad, Eficacia y Cambio en Educación, 14(2), 133-150. doi: http://doi.org/c2wx

Cejas, R. (2018). La formación en TIC del profesorado y su transferencia a la función docente. Tendiendo puentes entre tecnología, pedagogía y contenidos disciplinar. (Tesis Doctoral). Universidad Autónoma de Barcelona, Barcelona, España.

Centro de desarrollo del liderazgo educativo [CEDLE]. (2018). Aprendizaje para una política de formación de directores escolares en Chile. Informe de la política. Santiago, Chile: Centro de desarrollo del liderazgo educativo. Recuperado de https://goo.gl/gX3Jb4

Centro de Estudios del Ministerio de Educación [CEM]. (2013). Informe de resultados: Plan de formación de directores. Santiago, Chile: Centro de Estudios del Ministerio de Educación.

Centro de Perfeccionamiento, Experimentación e Investigación Pedagógica [CPEIP]. (2018). Síntesis estadística de los participantes del Plan de formación de directores 2011-2016. Documento de trabajo. Santiago, Chile: Centro de Perfeccionamiento, Experimentación e Investigación Pedagógica.

Centro Interamericano para el Desarrollo del Conocimiento en la Formación Profesional [OIT/Cinterfor]. (2011). Guía para la evaluación de impacto de la formación. Montevideo, Uruguay: Organización Internacional del Trabajo/Centro Interamericano para el Desarrollo del Conocimiento en la Formación Profesional. Recuperado de https://goo.gl/BLQ61H 
Cóndor-Quimbita, B., \& Remache-Bunci, M. (2019). La evaluación al desempeño directivo y docente como una oportunidad para mejorar la calidad educativa. Revista Cátedra, 2(1), 116-131. Recuperado de https://bit.ly/315RlQs

Consejo para la Transparencia. (2016). La evaluación de las políticas públicas, un elemento fundamental para la transparencia y la rendición de cuentas. Cuadernos de trabajo $N^{o}$ 5. Santiago, Chile: Consejo para la transparencia. Recuperado de https://goo.gl/BGvboJ Dirección de Presupuestos [DIPRES]. (2015). Evaluación ex-post: conceptos y metodologías. Santiago, Chile: Dirección de Presupuestos, Ministerio de Hacienda. Recuperado de https://goo.gl/iuGuv2

Escobar-Pérez, J., \& Cuervo-Martínez, A. (2008). Validez de contenido y juicio de expertos: una aproximación a su utilización. Avances en Medición, 6, 27-36.

Feinstein, O. (2007). Evaluación pragmática de políticas públicas. ICE, Revista de Economía, 1(836), 19-31. doi: https://doi.org/10.32796

Feixas, M., Fernández, A., Lagos, P., Quesada, C., \& Sabaté, S. (2013). Factores condicionantes de la transferencia de la formación docente en la universidad: un estudio sobre la transferencia de las competencias docentes. Infancia y Aprendizaje, 36(3), 401-416. doi: http://doi.org/c2wz

Feixas, M., Lagos, P., Fernández, I., \& Sabaté, S. (2015). Modelos y tendencias en la investigación sobre efectividad, impacto y transferencia de la formación docente en educación superior. Educar, 51(1), 81-107. doi: http://doi.org/c2zc

García-Garnica, M., \& Caballero, K. (2019). ¿La formación de los equipos directivos es suficiente para desempeñar prácticas eficaces de liderazgo pedagógico? Profesorado. Revista de Currículum y Formación de Profesorado, 23(2), 83- 106. doi: http://doi.org/c8xw

García-Martínez, I. \& Higueras-Rodríguez, L. (2018). La formación en el rol de la dirección eficaz en la comunidad autónoma de Andalucía. International Journal of Educational Leadership and Management, 6(2), 84-109. doi: http://doi.org/c8xx

García, M. (2003). El cuestionario como instrumento de investigación/evaluación. Documento de trabajo: Etapas del 
Proceso de Instrumentación. Extremadura, España: Centro Universitario Santa Ana. Recuperado de https://bit.ly/2Ket04d

Gertler, P., Martínez, S., Premand, P., Rawlings, L., \& Vermeersch, C. (2017). La evaluación de impacto en la práctica (Segunda edición). Washington, DC, EE.UU.: Banco Interamericano de Desarrollo y Banco Mundial. doi: http://doi.org/c22c

Gessler, M., \& Hinrichs, C. (2015). Key predictors of learning transfer in continuing vocational training. In Bohlinger, S., Haake, U., HelmsJørgensen, C., Toiviainen, H., y Wallo, A. (Eds.), Working and Learning in Times of Uncertainty (pp. 43-60). Rotterdam, Países Bajo: Sense Publishers. Recuperado de https://goo.gl/fZyu7y

Gilbert, A., \& Gibbs, G. (1999). A proposal for an international collaborative research programme to identify the impact of initial training on university teachers. Research and Development in Higher Education, 21, 131-143.

Gracia-Pérez, M., Gil-Lacruz, M., \& Gil-Lacruz, A. (2017). Evaluación del impacto de la modalidad formativa Learning by doing en profesionales sanitarios en Aragón. Contexto Educativos, 2, 27-45. doi: $\mathrm{http}: / / \mathrm{doi} . \mathrm{org} / \mathrm{dj} 7 \mathrm{~h}$

Hernández, R. (2018). Metodología de la investigación (2a ed.). México D.F.: McGraw-Hill.

Holton, E. (1996). The flawed four-level evaluation model. Human Resources Development Quarterly, 7(1), 5-21. Recuperado de https://goo.gl/PFGsCh

Kirkpatrick, D. (1999). Evaluación de las acciones formativas: Los cuatro niveles. Barcelona, España: Gestión 2000.

Kleinert, E., \& Pineda, P. (2018). La formación continua de docentes en educación infantil: El rol del directivo en la transferencia de aprendizajes. En F. Murillo (Coord.). Avances en democracia y liderazgo distribuido en educación: Actas del II Congreso Internacional de Liderazgo y Mejora de la Educación (p. 444-446). Red de Investigación sobre Liderazgo y Mejora de la Educación, Madrid, España. Recuperado de https://bit.ly/2Ks4YTF

López-Rodrigo, M., Feijoo-Cid, M., Novel-Martí, G., \& Leyva-Moral., J. (2017). Evaluación de la formación continuada. Análisis del proceso 
de transferencia y sostenibilidad. Atas-Investigação Qualitativa em Saúde, 2, 1165-1174. Recuperado de https://bit.ly/2Gc9nIq

Martínez, O., Domínguez, I., \& Infante, A. (2019). Indicadores para la evaluación del impacto de la formación laboral en la formación de los profesionales de la educación. Opuntia Brava, 11(2), 196-207. doi: http://doi.org/dj7f

Melo, R. (2014). Evaluación de la transferencia de programas de formación permanente del profesorado en la modalidad de asesoramiento: una propuesta hacia la evaluación de la transferencia. (Tesis Doctoral). Universidad de Barcelona, Barcelona, España.

Ministerio de Educación [MINEDUC]. (2016). Política de fortalecimiento del liderazgo directivo escolar. Chile: Ministerio de Educación. Recuperado de https://goo.gl/KESxBG

Ministerio de Educación de Chile [MINEDUC]. (2011). Decreto 44. Reglamentación de la ejecución del Plan de Formación de Directores. Chile: Ministerio de Educación. Recuperado de https://goo.gl/VBqFbk

Ministerio de Desarrollo Social [MDS]. (2015). Informe de seguimiento de programas sociales: Plan de formación de directores. Chile: Ministerio de Desasarrollo Social. Recuperado de https://bit.ly/2JxZSoK

Naquin, S., \& Holton, E. (2003). Motivation to improve work through learning in human resource development. Human Resource Development International, 6(3), 355-370. doi: http://doi.org/dfqrj4

Oficina Regional de Educación para América Latina y el Caribe [OREAL/UNESCO]. (2016). Recomendaciones de Políticas Educativas en América Latina en base al TERCE. Santiago, Chile: Oficina Regional de Educación para América Latina y el Caribe. Recuperado de https://goo.gl/AkkBtW

Park, J., \& Wentling, T. (2007). Factors associated with transfer of training in workplace elearning. Journal of Workplace Learning, 19(5), 311329. doi: http://doi.org/c74ff9

Pineda, P. (2000). La evaluación de la formación en las organizaciones: situación y perspectivas. Revista española de Pedagogía, 216, 291312. Recuperado de https://goo.gl/qvFmnE 
Pineda, P. (2011). Cómo medir el impacto de la formación: Un ejemplo del sector sanitario. Formación XXI, 18, 407-421. Recuperado de https://goo.gl/CFp7Dy

Ramos, F., Meizoso, M., \& Guerra, R. (2016). Instrumento para la evaluación del impacto de la formación académica. Revista Universidad y Sociedad, 8(2), 114-124. Recuperado de https://bit.ly/2Zo0mUy

Rent, A. (2013). La transferencia de aprendizaje en contextos de formación para el trabajo y el empleo. (Tesis doctoral). Universitat Rovira I Virgili, Tarragona, España.

Robinson, V., Hohepa, M., y Lloyd, C. (2009). School Leadership and Student Outcomes: Identifying what works and why. Best Evidence Synthesis Iteration [BES]. New Zeland: Ministry of Education. Recuperado de https://bit.ly/2SRLmvK

Tejada, J., \& Ferrández, E. (2007). La evaluación del impacto de la formación como estrategia de mejora en las organizaciones. REDIE. Revista Electrónica de Investigación Educativa, 9. Recuperado de https://goo.gl/gRRZXA

Tejada, J., Ferrández, E., Jurado, P., Más, O., Navío, A., \& Ruiz, C. (2008). Implicaciones de la evaluación de impacto: una experiencia de un programa de formación de formadores. Revista Bordón, 60(1), 163185. Recuperado de https://goo.gl/MmzxFg

Tomás-Folch, M., \& Duran-Bellonch, M. (2017). Comprendiendo los factores que afectan la transferencia de la formación permanente del profesorado. Propuestas de mejora. Revista Electrónica Interuniversitaria de Formación del Profesorado, 20(1), 145-157. doi: 10.6018/reifop.20.1.240591

Weinstein, J., \& Hernández, M. (2015). ¿Un centro nacional de liderazgo escolar en Chile? Antecedentes para su diseño. Documento de referencia $N^{\circ}$ 21. Santiago, Chile: Espacio Público. Recuperado de https://goo.gl/yfxBTc 
Richar Parra Robledo Profesor de ed. secundaria en Historia y Geografía, Magíster en Gestión directiva y Máster en Investigación educativa. Se ha desempeñado como coordinador académico en diferentes niveles escolares, sumado a consultorias y asesorías educativas. Actualmente cursa el doctorado en Educación en la Universidad Autónoma de Barcelona.

Contact Address: Carrer del Carme 1, 1, 1, Igualada (08700), España. E-mail: richar.parra.robledo@gmail.com

Carmen Ruiz Bueno. Profesora Titular del Departamento de Pedagogía Aplicada de la Universidad Autónoma de Barcelona, desde 02/05/2011. Doctora en Pedagogía, investigadora principal del grupo de investigación CIFO de la Universidad Autónoma de Barcelona. Desde 2001 se centra en el análisis de la Planificación, la evaluación y la innovación en el ámbito educativo. Las líneas de investigación se centran en las competencias docentes y su certificación, el diseño de acciones formativas, la innovación educativa, la formación de formadores, la docencia universitaria, y la evaluación de programas y el impacto de la formación. En la actualidad, los proyectos de investigación se centran en el desarrollo y la evaluación de competencias transversales en la enseñanza universitaria, concretamente en competencias vinculadas con el pensamiento reflexivo y crítico.

Contact Address: Departament de Pedagogia Aplicada, Edifici G6-246, Campus de Bellaterra, 08193, Bellaterra (Cerdanyola del Vallès)

E-mail: carmen.Ruiz.Bueno@uab.cat 